Pacific Journal of Mathematic 


\title{
ANALYTIC CONTINUATION OF MEROMORPHIC FUNCTIONS IN VALUED FIELDS
}

\author{
J. B. ROBERTS
}

In this paper* we consider analytic continuation of power series by matrix methods in arbitrary fields complete with respect to a valuation. In the complex field continuation can generally be achieved by a formal expansion of the given power series about a point in its circle of convergence. The new series (with power series coefficients) generally exists and converges over a circle extending beyond the circle of convergence of the original series.

When the field is non-Archimedean however the new circle of convergence is always contained in the old. Hence in this case we need have recourse to a summability method. In this paper we consider a certain class of matrix methods which can be applied to the power series coefficients appearing in the formal expansion of the original series about points outside the original circle of convergence. The methods will be applicable in Archimedean or non-Archimedean fields.

The work here is based upon Chapter 3 of the author's $\mathrm{PhD}$ dissertation written under the direction of Prof. G. K. Kalisch at the University of Minnesota in 1955.

1. Notations and definitions. Throughout the paper $k$ shall be a field which is complete with respect to a valuation, denoted by 11 . Unless stated explicitely to the contrary the valuation may be either Archimedean or non-Archimedean. It is useful to note that, by a theorem of Ostrowski, if the valuation is Archimedean then $k$ is topologically isomorphic with the real or complex numbers.

We shall designate the collection of all infinite series with terms in $k$ by $S$. Further we introduce an operation, the Cauchy product, into $S$. If $C=\sum_{i=0}^{\infty} c_{i}$ and $C^{\prime}=\sum_{i=0}^{\infty} c_{i}^{\prime}$ are in $S$ then the Cauchy product $C C^{\prime}$ is defined by

$$
C C^{\prime}=\sum_{i=0}^{\infty} \sum_{j=0}^{i} c_{j} c_{i-j}^{\prime} \text {. }
$$

This product is clearly in $S$; so $S$ is closed relative to this multiplication.

The subset of $S$ consisting of all unconditionally convergent series will be denoted by $T$. When $k$ is non-Archimedean $T$ coincides with the

* This paper was originally accepted by the Trans. Amer. Math. Soc., received by the editors of the Trans. Amer. Math. Soc. January 16, 1957, in revised form April 18, 1958. The author wishes to express his thanks to the referee who through his extensive comments on the first version has changed the character of the whole paper and has increased its generality in certain respects. 
set of all convergent series since in this case a series converges if and only if its $n$th term goes to 0 . When $k$ is Archimedean $T$ coincides with the collection of all absolutely convergent series. A theorem of Mertens in the Archimedean case (which remains true in the nonArchimedean case) assures us that $T$ is closed relative to the multiplication defined in $S$. Further by the same theorem if $C, C^{\prime}$ converge respectively to $c, c^{\prime}$ then $C C^{\prime}$ converges to $c c^{\prime}$.

The set of series in $T$ which converge to non-zero limits will be denoted by $T^{*}$. From the last sentence of the preceding paragraph we see that $T^{*}$ is closed under multiplication.

The set of infinite matrices $\left(a_{i j}\right), i=0,1,2, \cdots ; j=0,1,2, \cdots$ where $a_{i j}$ is in $k$ for all $i, j$ will be denoted by $M$. We introduce into $M$ two operations-addition and multiplication. Addition is unrestictedly defined by the following:

$$
m_{1}=\left(a_{i j}\right), m_{2}=\left(b_{i j}\right) \text { then } m_{1}+m_{2}=\left(a_{i j}+b_{i j}\right) .
$$

Clearly $m_{1}+m_{2}$ is in $M$.

Multiplication is not unrestrictedly defined. We have the following definition ( $m_{1}$ and $m_{2}$ as above):

$$
m_{1} m_{2}=\left(c_{i j}\right) \text { providing } c_{i j}=\sum_{q=0}^{\infty} a_{i q} b_{q j} \text { converges for all } i, j \text {. }
$$

We shall be interested in mappings from subsets of $S$ into $M$. A multiplicative homomorphism from a subset $V$ of $S$ into $M$ is a mapping $f$ of $V$ into $M$ such that when $C_{1}, C_{2}$ and $C_{1} C_{2}$ are in $V$ then $f\left(C_{1}\right) f\left(C_{2}\right)$ is defined and $f\left(C_{1}\right) f\left(C_{2}\right)=f\left(C_{1} C_{2}\right)$.

2. The matrices $A_{o}$ and $B_{\sigma}$.

Definition 1. Let $C=\sum_{i=0}^{\infty} c_{i}$ be in $S$.

(a) $B_{o}=\left(b_{i j}\right)$ where $b_{i j}=c_{j-i}$ and $c_{j-i}$ is taken to be 0 when $j<i$;

(b) If $C$ converges to $c \neq 0$ then $A_{\sigma}=B_{o}(c)^{-1}$ where $(c)$ is the diagonal matrix with all diagonal elements $c$.

Lemma 1. The map $C \rightarrow B_{0}$ is a multiplicative homomorphism of $S$ into $M$.

Proof. Let $C=\sum_{i=0}^{\infty} c_{i}, C^{\prime}=\sum_{i=0}^{\infty} c_{i}^{\prime}$ be in $S$. Then $C C^{\prime}=\sum_{i=0}^{\infty} \bar{c}_{i}$ where $\bar{c}_{i}=\sum_{j=0}^{i} c_{j} c_{i-j}^{\prime}$. Thus $B_{\sigma \sigma^{\prime}}=\left(\bar{c}_{j-i}\right)$. Since $B_{\sigma}$ and $B_{\sigma^{\prime}}$ each have only finitely many non-zero terms in each column $B_{\sigma} B_{\sigma^{\prime}}$ is defined. Further $B_{0} B_{\sigma^{\prime}}=\left(d_{i j}\right)$ where

$$
d_{i j}=\sum_{q=0}^{\infty} c_{q-i} c_{j-q}^{\prime}=\sum_{q=i}^{j} c_{q-i} c_{j-q}^{\prime}=\sum_{s=0}^{j-i} c_{s} c_{(j-i)-s}^{\prime}=\bar{c}_{j-i} .
$$


Hence $B_{\sigma} B_{\sigma^{\prime}}=B_{C C^{\prime}}$ and the lemma is proved.

Lemma 2. Let $C, C^{\prime}$ be in $S$ and suppose they converge respectively to the non-zero sums $c, c^{\prime}$. Then $A_{\sigma} A_{\sigma^{\prime}}$ exists and $A_{\sigma} A_{\sigma^{\prime}}=A_{\sigma^{\prime}}$ providing $C C^{\prime}$ converges to $c c^{\prime}$.

Proof. $A_{\sigma}=B_{\sigma}(c)^{-1}, A_{\sigma^{\prime}}=B_{\sigma^{\prime}}\left(c^{\prime}\right)^{-1}$ and therefore

$$
A_{\sigma} A_{\sigma^{\prime}}=B_{\sigma}(c)^{-1} B_{\sigma^{\prime}}\left(c^{\prime}\right)^{-1}=B_{\sigma \sigma^{\prime}}\left(c c^{\prime}\right)^{-1}=A_{\sigma \sigma^{\prime}} .
$$

Corollary. The map $C \rightarrow A_{\sigma}$ is a multiplicative homomorphism of $T^{*}$ into $M$.

We now introduce a norm into $T$ and two topologies into $M$.

Definition 2. The norm of $C$, denoted by $|C|_{r}$, for $C=\sum_{i=0}^{\infty} c_{i}$ in $T$ is defined by:

$$
|C|_{T}= \begin{cases}\max \left|c_{i}\right| & \text { for } k \text { non-Archimedean } \\ \sum_{i=0}^{\infty}\left|c_{i}\right| & \text { for } k \text { Archimedean }\end{cases}
$$

By restricting our $C$ to be in $T$ we insure that this norm is defined. The following properties are valid for arbitrary $k$.

$$
\begin{aligned}
& \left|C+C^{\prime}\right|_{T} \leq|C|_{T}+\left|C^{\prime}\right|_{T} ; \\
& \left|C C^{\prime}\right|_{T} \leq|C|_{T}\left|C^{\prime}\right|_{T} ; \\
& |a C|_{T}=|a||C|_{T} \text { for a in } k .
\end{aligned}
$$

If $k$ is non-Archimedean the first two properties can be strengthened to read

$$
\begin{aligned}
& \left|C+C^{\prime}\right|_{T} \leq \max \left(|C|_{T},\left|C^{\prime}\right|_{T}\right) ; \\
& \left|C C^{\prime}\right|_{T}=|C|_{T}\left|C^{\prime}\right|_{T} .
\end{aligned}
$$

Defining addition in $T$ to be componentwise addition we see that $T$ is a normed ring.

Definition 3. (a) The weak topology in $M$ is the topology induced on $M$ by making the sequence $m_{n}=\left(a_{i j}^{(n)}\right)$ of matrices converge to the matrix $\left(a_{i j}\right)$ if and only if for all $i, j$ we have $a_{i j}^{(n)} \rightarrow a_{i j}$. When this is true we say that the sequence $m_{n}$ converges weakly to $\left(a_{i j}\right)$.

(b) If, for an arbitrary positive real number $r$, we denote the set of all matrices $\left(a_{i j}\right)$ with $\left|a_{i j}\right|<r$, for all $i, j$, by $M_{r}$ then the set of $M_{r}$ gives a basis system for the open sets about the additive identity 0 in $M$. This induces on $M$ the topology of the additive group of $M$ and is called the uniform topology. 
We note that addition and multiplication (when the latter is defined) are continuous in both topologies in $M$. Also if a sequence of matrices converges in the uniform topology it converges in the weak topology.

We shall denote by $\bar{M}$ the collection of matrices $m=\left(a_{i j}\right)$ for which $\max \left|a_{i j}\right|$ exists. For $m$ in $\bar{M}$ we define $|m|=\max \left|a_{i j}\right|$. This induces the same topology on $\bar{M}$ which the uniform topology of $M$ induces on $\bar{M}$.

Lemma 3. The map $C \rightarrow B_{C}$ is a continuous map of $T$ into $M$ under either the uniform or weak topologies of: $M$.

Proof. Since $C$ is in $T, \max \left|c_{i}\right|$ exists and is $\leq|C|_{T}$. As $B_{C}=$ $\left(c_{j-i}\right)$ the norm of $B_{o}$, in $\bar{M}$, is given by

$$
\left|B_{o}\right|=\max \left|c_{j-1}\right|=\max \left|c_{i}\right| \leq|C|_{T} \text {. }
$$

Therefore the map of $T$ into $\bar{M}$ is continuous with respect to the norm topology of $\bar{M}$. Since this topology is induced by the uniform topology of $M$ this map is continuous relative to the uniform topology. This then implies continuity relative to the weak topology and the lemma is proved.

Lemma 4. The $\operatorname{map} C \rightarrow A_{0}$ is a continuous $\operatorname{map}$ of $T^{*}$ into $M$ under either the uniform or weak topologies of $M$.

Proof. $A_{\sigma}=B_{\sigma}(c)^{-1}$ where $c \neq 0$ is the sum of $C$. Since multiplication in $M$ is continuous in either topology as is the map $C \rightarrow B_{0}$ (by previous lemma) we need only show that the map $C \rightarrow(c)^{-1}$ is continuous. This is the product of three maps $C \rightarrow c \rightarrow c^{-1} \rightarrow(c)^{-1}$.

The first is continuous since it is an additive homomorphism and $|c| \leq$ $|C|_{T}$. The second is a continuous map on $k^{*}$ (the non-zero elements of $k$ ). The third map is a ring isomorphism into $\bar{M}$ preserving norms. I.e. $\left|c^{-1}\right|=\max \left|c^{-1}\right|=\left|\left(c^{-1}\right)\right|$. Hence this map is continuous into $\bar{M}$ relative to the norm in $\bar{M}$. As in the proof of Lemma 3 this concludes the proof.

We define the convergence of an infinite product $\prod_{n=1}^{\infty} C_{n}, C_{n}$ in $T$, in the usual way. That is, $\prod_{n=1}^{\infty} C_{n}$ converges providing $\lim _{q \rightarrow \infty} \prod_{n=1}^{q} C_{n}$ exists and is not the additive identity of $T$. Making use of the theorem: $\prod_{n=1}^{\infty} C_{n}, C_{n}$ in $T$, converges if and only if $\left|1-C_{n}\right|_{T} \rightarrow 0$ as $n \rightarrow \infty$ (where 1 is the multiplicative identity in $T$ ).

We deduce from Lemma 4 the following immediate consequence.

THEOREM 1. Let $\sum_{n=1}^{\infty} C_{n}$ converge and suppose $C_{n}$ is in $T^{*}$ for all n. Then $\prod_{n=1}^{\infty} A_{o_{n}}$ converges relative to both weak and uniform topologies of $M$ and its limit is $A_{\prod_{n=1}^{\infty} C_{n}}$. 
3. $T_{2}$ matrices and $C(x)$-continuation. Each infinite matrix $m$ can be thought of as a mapping defined over a subset of $S$ and mapping this subset into $S$. In fact, let $m=\left(a_{i j}\right)$ and suppose $C=\sum_{i=0}^{\infty} c_{i}$ is in $S$. Then if, for all $j$, the series $\sum_{i=0}^{\infty} c_{i} a_{i j}$ exists and equals $c_{j}^{\prime}$ we shall say that the matrix $m$ maps $C$ onto $C^{\prime}=\sum_{j=0}^{\infty} c_{j}^{\prime}$. We shall write $m C=C^{\prime}$.

(If we let $C^{*}$ be the "vector" $\left(c_{0}, c_{1}, c_{2}, \cdots\right)$ derived in the obvious way from $C$ then $C^{\prime *}=(m C)^{*}=C^{*} \cdot m$ where the right side is the ordinary matrix product of $C^{*}$ and $m$.)

When $C$ has sum $c$ then $C^{\prime}$ has sum $c$ we call $m$ a $T_{2}$ matrix. Necessary and sufficient conditions in order that an infinite matrix be a $T_{2}$ matrix will be found in [2] for $k$ Archimedean and in [8ab] for $k$ non-Archimedean. (In the reference [2] the $T_{2}$ matrix is called an $\alpha$ matrix.)

Now suppose $C=\sum_{i=0}^{\infty} c_{i}, C^{\prime}=\sum_{i=0}^{\infty} c_{i}^{\prime}$ are in $T$ with sums $c, c^{\prime}$ respectively. Then $C C^{\prime}$ exists and

$$
C C^{\prime}=\sum_{j=0}^{\infty} \sum_{i=0}^{j} c_{i}^{\prime} c_{j-i}=B_{o} C^{\prime}
$$

where $B_{0}$ is as defined in $\S 2$.

Since in this case $C C^{\prime}$ converges to $c c^{\prime}$ we see that $B_{\sigma}$ maps convergent series onto convergent series but alters the sum by a factor of c. Thus for $C$ in $T^{*}, A_{o}=B_{o}(c)^{-1}$ will map convergent series onto convergent series with the same sum. This proves the following.

\section{Lemma 5. If $C$ is in $T^{*}$ then $A_{\sigma}$ is a $T_{2}$ matrix.}

We wish now to consider series of functions. Let $C(x)=\sum_{i=0}^{\infty} c_{i}(x)$ and $U(x)=\sum_{i=0}^{\infty} u_{i}(x)$ where $x$ ranges over some subset $X$ of $k$. Suppose in addition that $C(x)$ is in $T^{*}$ for all $x$ in $D$ where $D$ is a subset of $X$. Further suppose there is a non-empty subset $\Delta$ of $D$ on which $U(x)$ converges. Then, by Lemma $5, A_{C(x)}$ is a $T_{2}$ matrix for $x$ in $D$ and therefore transforms $U(x)$, for $x$ in $\Delta$, into a new series with the same sum. However it may be true that $A_{\sigma(x)} U(x)$ is defined and converges for some $x$ in $D-\Delta$.

The sum function $u^{\prime}(x)$, considered over the largest portion of $D$ on which $A_{\sigma(x)} U(x)$ exists and converges, will be called the $C(x)$-continuation of $U(x)$ (or more accurately the $C(x)$-continuation of the sum function $u(x)$ of $U(x))$. The $C(x)$-continuation will be called efficient for $U(x)$ if there exists an $x$ in $D-\Delta$ for which $A_{\sigma(x)}$ exists and converges.

In Archimedean fields it is possible for an infinite series to converge conditionally. If $C(x)$ converges conditionally for some $x$ then $A_{\sigma(x)}$ is defined but is not necessarily a $T_{2}$ matrix (since the Cauchy product of conditionally convergent series may not converge). Considering $X$ now 
to be a topological space we can speak of the closure $\bar{D}$ of $D$ in $X$. Let $x$ be in $\bar{D}$ and suppose that when $y$ in $D$ converges to $x$ in certain prescribed ways then $C(x), U(x), A_{C(x)} U(x)$ converge respectively to

$$
\lim _{y \rightarrow x} c(y), \lim _{y \rightarrow x} u(y), \lim _{y \rightarrow x} u^{\prime}(y)
$$

when these limits exist. When $x$ is in $D$ we know $u(x)=u^{\prime}(x)$ so $A_{\sigma(x)} U(x)$ converges when $U(x)$ does. Thus if $c(x), u(x), u^{\prime}(x)$ are continuous over $D$ then for $x$ in $D$, whatever the prescribed ways $y$ in $D$ tends to $x$, we have $C(x), U(x), A_{C(x)}, U(x)$ converging respectively to

$$
\lim _{y \rightarrow x} c(y), \lim _{y \rightarrow x} u(y), \lim _{y \rightarrow x} u^{\prime}(y) .
$$

Let $D^{*}$ be the set of all $x$ in $\bar{D}$ for which $C(x), U(x), A_{\sigma(x)} U(x)$ have the respective limits specified above as $y \rightarrow x$ in one of the prescribed ways. Then $D \subset D^{*} \subset \bar{D}$ when $c(x), u(x), u^{\prime}(x)$ are continuous over $D$. The function $u^{\prime}(x)$, considered over $D^{*}$, will be called a generalized $C(x)$-continuation of $U(x)$ (relative to the allowed modes of convergence of $y$ in $D$ to $x$ in $D^{*}$ ).

4. Power series and the Weierstrass decomposition theorem. In this section we take the $X$ of $\S 3$ to be all of $k$ and suppose $C(x)$ and $U(x)$ to be power series about $\alpha$ in $k$. Then we may take, without loss of generality, the set $D$ to be a circle with center $\alpha$ from which have been excised all zeros of $C(x)$. Then $\Delta$ is the intersection of $D$ with some circle of center $\alpha$. When $k$ is non-Archimedean $\bar{D}=D$ and when $k$ is Archimedean $\bar{D}$ is the closed circle about $\alpha$ of the same radius as $D$. Thus (by Abel's theorem in the Archimedean case) if we prescribe $y$ in $D$ to converge to $x$ in $\bar{D}-D$ only radially we can take $D^{*}=\bar{D}$.

Theorem 2. For $x_{0} \neq 0, n=0,1,2, \cdots$ let $C_{n}\left(x_{0}\right)=\sum_{i=0}^{\infty} a_{n i} x_{0}^{i}$ and $C\left(x_{0}\right)=\sum_{i=0}^{\infty} c_{i}\left(x_{0}\right)$ be in $T$. Then if $C_{n}\left(x_{0}\right) \rightarrow C\left(x_{0}\right)$ the following are true.

(i) for each $i$ there is an $a_{i}$ such that $\lim _{n \rightarrow \infty} a_{n i}=a_{i}$;

(ii) $a_{i} x_{0}^{i}=c_{i}\left(x_{0}\right)$.

That is, $C\left(x_{0}\right)$ is the term by term limit of $C_{n}\left(x_{0}\right)$ when it is the limit in the $T$ norm.

Proof. Since $\left|C_{n}\left(x_{0}\right)-C\left(x_{0}\right)\right|_{T} \rightarrow 0$ we have $\left|C_{n}\left(x_{0}\right)-C_{m}\left(x_{0}\right)\right|_{T} \rightarrow 0$ as $n, m \rightarrow \infty$ independently. But

$$
\left|C_{n}\left(x_{0}\right)-C_{m}\left(x_{0}\right)\right|_{T}=\left|\sum_{i=0}^{\infty}\left(a_{n i}-a_{m i}\right) x_{0}^{i}\right|=\left\{\begin{array}{l}
\max _{i}\left|a_{n i}-a_{m i}\right|\left|x_{0}\right|^{i} \\
\sum_{i=0}^{\infty}\left|a_{n i}-a_{m i}\right|\left|x_{0}\right|^{i}
\end{array}\right.
$$

in the cases where $k$ is non-Archimedean or Archimedean respectively. In either event $\left|a_{n i}-a_{m i}\right|\left|x_{0}\right|^{i} \leq\left|C_{n}\left(x_{0}\right)-C_{m}\left(x_{0}\right)\right|_{r} \rightarrow 0$. Hence by com- 
pleteness of $k$ there is an $a_{i}$ such that $a_{n i} \rightarrow a_{i}$. This proves (i). To prove (ii) we have

$$
\begin{aligned}
\left|a_{i} x_{0}^{i}-c_{i}\left(x_{0}\right)\right| & \leq\left|a_{i} x_{0}^{i}-a_{n i} x_{0}^{i}\right|+\left|a_{n i} x_{0}^{i}-c_{i}\left(x_{0}\right)\right| \\
& \leq\left|a_{i} x_{0}^{i}-a_{n i} x_{0}^{i}\right|+\left|C_{n}\left(x_{0}\right)-C\left(x_{0}\right)\right|_{T} .
\end{aligned}
$$

Since both terms on the right tend to zero the proof of (ii) is completed.

We now suppose that $k$ is algebraically closed and is non-Archimedean. If $C(x)=\sum_{i=0}^{\infty} a_{i} x^{i}$ is an entire power series (i.e. $C(x)$ is in $T$ for all $x$ in $k$ ) which is not identically zero then by the analogue of the Weierstrass decomposition theorem in algebraically closed non-Archimedean fields (see Schöbe [10] and Schnirelman [11]) we can express $C(x)$ as the formal limit of

$$
a_{0} x^{i} \prod_{q} \prod_{q=0}^{n \leq \infty}\left(1-x / z_{q}\right)
$$

where $i_{0}$ is the multiplicity of the zero $x=0$ of the sum $c(x)$ of $C(x)$ and where $z_{q}$ ranges over the set of non-zero zeros of $c(x)$, each factor $1-x / z_{q}$ occuring a number of times equal to the multiplicity of $z_{q}$ as a zero of $c(x)$.

Schöbe [10] has also proved that $\left|z_{q}\right| \rightarrow \infty$ as $q \rightarrow \infty$. Therefore, since the terms of the product are power series and $1+\left(1-x / z_{q}\right)=$ $x / z_{q}$ has $\left|x / z_{q}\right|_{T} \rightarrow 0$, the product $\prod_{q=0}^{n \leq \infty}\left(1-x / z_{q}\right)$, when infinite, converges for every $x$ in $k$, relative to the topology of $T$. Hence by Theorem 2 above this product converges to $C(x)$. These remarks combined with Theorem 1 prove the following theorem. The notation is as above.

Theorem 3. Let $C(x)$ be an entire power series. Then for $x$ a nonzero of the sum function $c(x)$ of $C(x)$ we have

$$
A_{O(x)}=A_{a_{0}} A_{x}^{i} \prod_{q=0}^{n \leq \infty} A_{1-x / z_{q}}
$$

where, if $n$ is infinite, the right side converges to the left in both the uniform and weak topologies of $M$.

In the case of the complex field the original Weierstrass decomposition theorem gives an analogous result where the $A_{1-x / z_{q}}$ are replaced by more complicated matrices corresponding to the primary factors of $C(x)$.

5. Meromorphic functions and $C(x)$-continuation. If the function $f(x)$ has a Taylor series expansion $\sum_{i=0}^{\infty} a_{i}(x-\alpha)^{i}$ about $\alpha$ in $k$ which converges to $f(x)$ in its circle of convergence we shall denote this series by $[f(x)]_{x}$. If $D$ is the circle of convergence and $y$ is an interior point of $D$ we can expand $[f(x)]_{x}$ about $y$ to obtain (formally) $[f(x)]_{y}$. Thus 


$$
[f(x)]_{y} \sim \sum_{i=0}^{\infty} \sum_{j=0}^{\infty}\left(\begin{array}{c}
i+j \\
i
\end{array}\right) a_{i+j}(y-\alpha)^{j}(x-y)^{i} .
$$

We shall denote $\sum_{j=0}^{\infty}\left(\begin{array}{c}i+j \\ i\end{array}\right) a_{i+j}(y-\alpha)^{j}$ by $\left[f_{i}(y)\right]_{x}$. When the charracteristic of $k$ is 0 we know

$$
\left[f_{i}(y)\right]_{\alpha}=(1 / i !)\left[\frac{d^{i} f(y)}{d y^{i}}\right]_{\alpha} .
$$

(Although much of what we shall say is true for fields of arbitrary characteristic we confine ourselves to fields of characteristic 0 in order to simplify the discussion.)

Letting $f_{i}(y)$ be the sum function of $\left[f_{i}(y)\right]_{\alpha}$ we have

$$
[f(x)]_{y}=\sum_{i=0}^{\infty} f_{i}(y)(x-y)^{i} .
$$

It is known that in both the Archimedean and non-Archimedean case that for all $i,\left[f_{i}(y)\right]_{\alpha}$ converges for all $y$ in $D$. However in the Archimedean case it is often true that there is a circle $D_{1}$, not contained wholly within $D$, and in which, for all $i,\left[f_{i}(y)\right]_{x}$ converges and $\sum_{i=0}^{\infty}\left[f_{i}(y)\right](x-y)^{i}$ converges in $D_{1}$ to $f(x)$.

This allows one to step by step recover the function $f(x)$ from a power series element $[f(x)]_{x}$ of the function. When $k$ is non-Archimedean it can be shown that no such circle as $D_{1}$ can ever exist. Thus the usual method of analytic continuation necessarily fails in such fields. In this section we shall show how $C(x)$-continuation can be applied in the case of the continuation of power series elements of meromorphic functions with known denominators (see below).

Let $D$ be a circle in $k$ ( $D$ open if $k$ is Archimedean) with center $\alpha$. A function $f(x)$ defined over some subset of $k$ will be side to be meromorphic over $D$ if there exist two series $[g(x)],[h(x)]_{x}$ convergent on $D$ such that

(i) $f(x)$ is defined for $x$ in $D$ if and only if $h(x) \neq 0$;

(ii) $f(x)=g(x) / h(x)$ everywhere on $D$ where defined. The function $h(x)$ will be called a denominator of $f(x)$ over $D$. If $D$ is the greatest such circle we call it the circle of meromorphy of $f(x)$.

Lemma 6. If $f(x)$ is meromorphic on $D$ with denominator $h(x)$ and if $\alpha$ is in $D$ then $f(x)$ is the $[h(x)]_{\alpha}$-continuation of $[f(x)]_{x}$.

Proof. Let $x$ be in $D, h(x) \neq 0$. Then there is a $g(x)$ such that $[g(x)]_{x}$ converges on $D$ and $f(x)=g(x) / h(x)$. Now $f(x)$ is the sum function of 


$$
[g(x)]_{\alpha} / h(x)=\left([h(x)]_{\alpha} / h(x)\right)[f(x)]_{x}=A_{[h(x)]_{\alpha}}[f(x)]_{x}
$$

and the lemma is proved.

THEOREM 4. Let $f(x)$ be meromorphic on $D$ with denominator $h(x)$. Further for $\alpha$ in $D$ let

$$
[f(x)]_{x} \sum_{i=0}^{\infty} a_{i}(x-\alpha)^{i}
$$

have circle of convergence contained in $D$. Then

$$
[f(x)]_{y}=\sum_{i=0}^{\infty} f_{i}(y)(x-y)^{i}
$$

when $y$ is in $D, h(y) \neq 0$, and $f_{i}(y)$ is the $[h(y)]_{\alpha}^{i+1}$-continuation of $i ! \sum_{j=0}^{\infty}\left(\begin{array}{c}i+j \\ i\end{array}\right) a_{i+j}(y-\alpha)^{j}$.

Proof. As seen above the formal expansion of $[f(x)]_{\alpha}$ about $y$ is given by

$$
\sum_{i=0}^{\infty} \sum_{j=0}^{\infty}\left(\begin{array}{c}
i+j \\
i
\end{array}\right) a_{i+j}(y-\alpha)^{j}(x-y)^{i}
$$

where

$$
i ! \sum_{j=0}^{\infty}\left(\begin{array}{c}
i+j \\
i
\end{array}\right) a_{i+\jmath}(y-\alpha)^{j}=\left[\frac{d^{i} f(y)}{d y^{i}}\right]_{\alpha} .
$$

But

$$
\frac{d^{i} f(y)}{d y^{i}}=\frac{d^{i}(g(y) / h(y))}{d y^{i}}=t(y) / h^{i+1}(y)
$$

where $t(y)$ is a polynomial in $g(y)$ and $h(y)$. Thus $[t(y)]_{\alpha}$ converges over $D$ and $d^{i} f(y) / d y^{i}$ is meromorphic on $D$ with denominator $(t(y))^{i+1}$. Thus by Lemma $1, d^{i} f(y) / d y^{i}$ is the $\left[(h(y))^{i+1}\right]_{\alpha}$-continuation of

$$
\left[d^{i} f(y) / d y^{i}\right]_{\alpha}=i ! \sum_{j=0}^{\infty}\left(\begin{array}{c}
i+j \\
i
\end{array}\right) a_{i+j}(y-\alpha)^{j} .
$$

From Theorem 4 and Theorem 1 we have the

Corollary. $f_{i}(y)$ is the sum function of $A_{[n(y)]_{a}}^{i+1}\left[d^{i} f(y) / d y^{i}\right]_{x}$.

THEOREM 5. If a function $f(x)$ defined over $D$ is the $C(x)$-continuation of a power series $[f(x)]_{\infty}$ then $f(x)$ is meromorphic on $D$ when $C(x)$ is of the form $[h(x)]_{\alpha}$. 
Proof. Let $f(x)$ be the sum function of

$$
A_{[h(x)]_{\alpha}}[f(x)]_{\alpha}=\left([h(x)]_{\alpha} / h(x)\right)[f(x)]_{\alpha}
$$

which converges on $D$. Then $[g(x)]_{\alpha}=[h(x)]_{\alpha}[f(x)]_{\alpha}$ converges on $D$. Letting $g(x), h(x)$ be the sums on $D$ of $[g(x)]_{\alpha},[h(x)]_{x}$ respectively gives $[f(x)]_{\alpha}$ the expansion about $\alpha$ of $g(x) / h(x)$ which is meromorphic on $D$.

There are many further questions which can be asked concerning these methods of continuation. In view of Theorem 5 one would wish to concentrate on $C(x)$-continuations where $C(x)$ is not a power series.

Further we can generalize the method so that instead of restricting ourselves to the use of $C(x)$-continuations we allow the use of arbitrary $T_{2}$ matrices. Some work has been done in this direction in [8a].

Vermes, making use of series to sequence methods, has dealt with similar problems for $k$ the field of complex numbers [13abc]. Some of his results in [13a] overlap some of the work done here. For further considerations of these and similar problems see the references to Chabauty, Krasner, Kürshák, Rychlik, Schöbe and Strassman cited below.

\section{REFERENCES}

1. C. Chabauty, Sur la théorie des fonctions dans un corp valué I, II, C.R. Acad. Sci., Paris, 231, (1950), 396-7, 432-4.

2. R. G. Cooke, Infinite Matrices and Sequence Spaces, Macmillan, (1950).

3. J. Hadamard, Essai sur l'étude des fonctions données par leur développement de Taylor, J. Math. 4, (1892), 8.

4. H. Hasse, Zahlentheorie, Akademie-Verlag, Berlin, (1949).

5. M. Krasner, (a) Essai d'une théorie des fonctions analytiques dans les valués complets, C. R. Acad. Sci., Paris 222, (1946), 37-40, 165-7, 363-5, 581-3.

(b). Certaines propriśtés des séries de Taylor d'un ensemble au plus dénombrable de variables dans les corps valués complets et une demonstration structurale des formules de M. Pollaczek, Bull. des Sci. Mathématique 71, (1947), 123-152, 180-200.

(c) Prolongement analytique dans les corps valués complets C. R. Acad. Sci. Paris 238, (1954), 2385-7 239, (1954), 468-470, 745-7, 244, (1957), 1304-6, 1599-1602, 1996-9, 2510-2573, 245, (1957), 1285-8.

6. J. Kürschák, Limesbildung und allgemeine Körpertheorie, J. reine angew. Math. 142, (1913), 211-253.

7. F. Loonstra, Analytische Untersuchungen über bewertete Körper N.V. Noord-Hollandisch Uitgevers Maatschappij, Amsterdam (1941).

8. J. B. Roberts, (a) Summability methods in valuation fields, Thesis, Univ. of Minnesota (1955).

(b) Matrix summability in F-fields, Proc. Amer. Math. Soc. 8, (1957) 541-3.

9. K. Rychlik, Zur Bewertungstheorie der algebraischen Körper, J. reine angew. Math. 153, (1924) 94-107.

10. W. Schöbe, Beiträge zur Functionentheorie in nicht-archimidisch bewerteten Körper n, Universitas-Archiv, Band 42 des Archiv, Band 2 der Abteilung (1950).

11. L. Schnirelman, Functions in algebraically closed valuation flelds (in Russian), Burl. Acad. Sci. URSS, Serié Math., 5, 6, (1938), 487-498.

12. R. Strassman, Uber den Wertevorrat von Potenzreihen im Gebiet der p-adischen Zahten, J. reine angew. Math. 159, (1928) 13-28. 
13. P. Vermes, (a) On $\gamma$-matrices and their application to the binomial series, Proc. Ed. Math. Soc. 3, 8, (1947), 1-13.

(b) The application of $\gamma$-matrices to Taylor series, Proc. Ed. Math. Soc. 2, 8, (1948), 43-9

(c) Series to series transformations and analytic continuation by matrix melhods Amer. J. Math. 71 (1949), 541-562.

\section{REED COLLEGE}





\section{PACIFIC JOURNAL OF MATHEMATICS}

\section{EDITORS}

\section{David Gilbarg}

Stanford University

Stanford, California

R. A. Beaumont

University of Washington

Seattle 5 , Washington
A. L. Whiteman

University of Southern California

Los Angeles 7, California

L. J. PAIGE

University of California

Los Angeles 24, California

\section{ASSOCIATE EDITORS}
E. F. BECKENBACH
C. E. BURGESS
E. HEWITT
A. HORN

\author{
V. GANAPATHY IYER \\ R. D. JAMES \\ M. S. KNEBELMAN \\ L. NACHBIN
}
I. NIVEN
E. G. STRAUS
T. G. OSTROM
H. L. ROYDEN
G. SZEKERES
M. M. SCHIFFER
F. WOLF
K. YOSIDA

\section{SUPPORTING INSTITUTIONS}

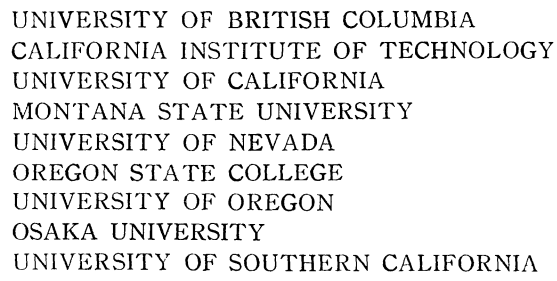

UNIVERSITY OF BRITISH COLUMBIA CALIFORNIA INSTITUTE OF TECHNOLOGY UNIVERSITY OF CALIFORNIA MONTANA STATE UNIVERSITY UNIVERSITY OF NEVADA OREGON STATE COLLEGE UNIVERSITY OF OREGON OSAKA UNIVERSITY UNIVERSITY OF SOUTHERN CALIFORNIA

STANFORD UNIVERSITY

UNIVERSITY OF TOKYO

UNIVERSITY OF UTAH

WASHINGTON STATE COLLEGE

UNIVERSITY OF WASHINGTON

AMERICAN MATHEMATICAL SOCIETY CALIFORNIA RESEARCH CORPORATION HUGHES AIRCRAFT COMPANY SPACE TECHNOLOGY LABORATORIES

Printed in Japan by Kokusai Bunken Insatsusha (International Academic Printing Co., Ltd.), Tokyo, Japan 


\section{Pacific Journal of Mathematics}

\section{Vol. 9, No. 1 \\ May, 1959}

Julius Rubin Blum and Murray Rosenblatt, On the structure of infinitely

divisible distributions . ............................. 1

Robert Geroge Buschman, Asymptotic expressions for

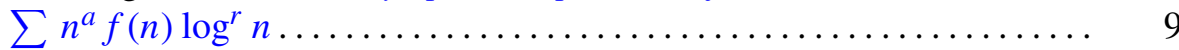

Eckford Cohen, A class of residue systems $(\bmod r)$ and related arithmetical

functions. I. A generalization of Möbius inversion .............. 13

Paul F. Conrad, Non-abelian ordered groups ................... 25

Richard Henry Crowell, On the van Kampen theorem............... 43

Irving Leonard Glicksberg, Convolution semigroups of measures ........ 51

Seymour Goldberg, Linear operators and their conjugates ............ 69

Olof Hanner, Mean play of sums of positional games .............. 81

Erhard Heinz, On one-to-one harmonic mappings ................ 101

John Rolfe Isbell, On finite-dimensional uniform spaces . . ........... 107

Erwin Kreyszig and John Todd, On the radius of univalence of the function

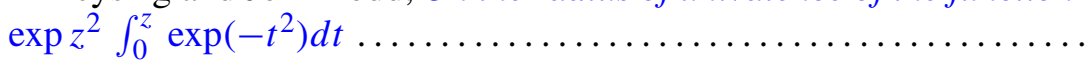

Roger Conant Lyndon, An interpolation theorem in the predicate

calculus......................................... 129

Roger Conant Lyndon, Properties preserved under homomorphism ........ 143

Roger Conant Lyndon, Properties preserved in subdirect products ....... 155

Robert Osserman, A lemma on analytic curves ................ 165

R. S. Phillips, On a theorem due to Sz.-Nagy..................... 169

Richard Scott Pierce, A generalization of atomic Boolean algebras ....... 175

J. B. Roberts, Analytic continuation of meromorphic functions in valued fields................................. 183

Walter Rudin, Idempotent measures on Abelian groups ................ 195

M. Schiffer, Fredholm eigen values of multiply-connected domains ........ 211

V. N. Singh, A note on the computation of Alder's polynomials ......... 271

Maurice Sion, On integration of 1-forms ...................... 277

Elbert A. Walker, Subdirect sums and infinite Abelian groups........... 287

John W. Woll, Homogeneous stochastic processes . .................. 293 